\title{
UNIQUENESS OF SOLUTIONS FOR A NONLOCAL ELLIPTIC EIGENVALUE PROBLEM
}

\author{
Craig Cowan And Mostafa Fazly
}

AbStract. We examine equations of the form

$$
\begin{cases}(-\Delta)^{\frac{1}{2}} u=\lambda g(x) f(u) & \text { in } \Omega \\ u=0 & \text { on } \partial \Omega\end{cases}
$$

where $\lambda>0$ is a parameter and $\Omega$ is a smooth bounded domain in $\mathbb{R}^{N}, N \geq 2$. Here $g$ is a positive function and $f$ is an increasing, convex function with $f(0)=1$ and either $f$ blows up at 1 or $f$ is superlinear at infinity. We show that the extremal solution $u^{*}$ associated with the extremal parameter $\lambda^{*}$ is the unique solution. We also show that when $f$ is suitably supercritical and $\Omega$ satisfies certain geometrical conditions then there is a unique solution for small positive $\lambda$.

\section{Introduction}

We are interested in the following nonlocal eigenvalue problem

$$
(P)_{\lambda} \quad \begin{cases}(-\Delta)^{\frac{1}{2}} u=\lambda g(x) f(u) & \text { in } \Omega \\ u=0 & \text { on } \partial \Omega,\end{cases}
$$

where $(-\Delta)^{\frac{1}{2}}$ is the square root of the Laplacian operator, $\lambda>0$ is a parameter, $\Omega$ is a smooth bounded domain in $\mathbb{R}^{N}$ where $N \geq 2$, and where $0<g(x) \in C^{1, \alpha}(\bar{\Omega})$ for some $0<\alpha$. The nonlinearity $f$ satisfies one of the following two conditions:

(R) $\quad f$ is smooth, increasing and convex on $\mathbb{R}$ with $f(0)=1$ and $f$ is superlinear at $\infty$ (i.e., $\lim _{t \rightarrow \infty} \frac{f(t)}{t}=\infty$ ), or

(S) $\quad f$ is smooth, increasing, convex on $[0,1)$ with $f(0)=1$ and $\lim _{t / 1} f(t)=+\infty$.

In this paper, we prove there is a unique solution of $(P)_{\lambda}$ for two parameter ranges: for small $\lambda$ and for $\lambda=\lambda^{*}$ where $\lambda^{*}$ is the so called extremal parameter associated with $(P)_{\lambda}$. First, let us to recall various known facts concerning the second order analog of $(P)_{\lambda}$.

Some notations: Let $F(t):=\int_{0}^{t} f(\tau) d \tau$ and $C_{f}:=\int_{0}^{a_{f}} f(t)^{-1} d t$ where $a_{f}=\infty$ (resp. $a_{f}=1$ ) when $f$ satisfies $(\mathrm{R})$ (resp. $f$ satisfies $(\mathrm{S})$ ). We say a positive function $f$ defined on an interval $I$ is logarithmically convex (or log convex) provided $u \mapsto$ $\log (f(u))$ is convex on $I$. Also, $\Omega$ will always denote a smooth bounded domain in $\mathbb{R}^{N}$ where $N \geq 2$.

Received by the editors September 23, 2011.

1991 Mathematics Subject Classification. xxx.

Key words and phrases. xxx.

The second author is partially supported by a University Graduate Fellowship and this work is part of his Ph.D. dissertation in preparation under the supervision of N. Ghoussoub. 
1.1. The local eigenvalue problem. For a nonlinearity $f$ which satisfies $(\mathrm{R})$ or $(\mathrm{S})$, the following second order analog of $(P)_{\lambda}$ with the Dirichlet boundary conditions

$$
(Q)_{\lambda} \quad \begin{cases}-\Delta u=\lambda f(u) & \text { in } \Omega \\ u=0 & \text { on } \partial \Omega,\end{cases}
$$

is by now quite well understood whenever $\Omega$ is a bounded smooth domain in $\mathbb{R}^{N}$. See, for instance, $[2-5,14-16,18,20,21]$. We now list the properties one comes to expect when studying $(Q)_{\lambda}$.

It is well known that there exists a critical parameter $\lambda^{*} \in(0, \infty)$ such that for all $0<\lambda<\lambda^{*}$ there exists a smooth, minimal solution $u_{\lambda}$ of $(Q)_{\lambda}$. Here the minimal solution means in the pointwise sense. In addition for each $x \in \Omega$ the map $\lambda \mapsto$ $u_{\lambda}(x)$ is increasing in $\left(0, \lambda^{*}\right)$. This allows one to define the pointwise limit $u^{*}(x):=$ $\lim _{\lambda / \lambda^{*}} u_{\lambda}(x)$ which can be shown to be a weak solution, in a suitably defined sense, of $(Q)_{\lambda^{*}}$. It is also known that for $\lambda>\lambda^{*}$ there are no weak solutions of $(Q)_{\lambda}$. Also, one can show that the minimal solution $u_{\lambda}$ is a semi-stable solution of $(Q)_{\lambda}$ in the sense that

$$
\int_{\Omega} \lambda f^{\prime}\left(u_{\lambda}\right) \psi^{2} \leq \int_{\Omega}|\nabla \psi|^{2}, \quad \forall \psi \in H_{0}^{1}(\Omega) .
$$

We now come to the results known for $(Q)_{\lambda}$ that we are interested in extending to $(P)_{\lambda}$. In [18] it was shown that the extremal solution $u^{*}$ is the unique weak solution of $(Q)_{\lambda^{*}}$. Some of the techniques involve using concave cut offs which do not seem to carry over to the nonlocal setting. Here, we use some techniques developed in [1] that were used in studying a fourth order analogue of $(Q)_{\lambda}$. In [11] the uniqueness of the extremal solution for $\Delta^{2} u=\lambda e^{u}$ on radial domains with Dirichlet boundary conditions was shown and this was extended to log convex (see below) nonlinearities in [17]. Some of the methods used in [17] were inspired by the techniques of [1] and so will ours in the case where $f$ satisfies (R). In [8] it was shown that the extremal solution associated with $\Delta^{2} u=\lambda(1-u)^{-2}$ on radial domains is unique and our methods for nonlinearities satisfying $(\mathrm{S})$ use some of their techniques.

In [19] and [23] a generalization of $(Q)_{\lambda}$ was examined. They showed that if $f$ is suitably supercritical at infinity and if $\Omega$ is a star-shaped domain, then for small $\lambda>0$ the minimal solution is the unique solution of $(Q)_{\lambda}$. In [13] this was done for a particular nonlinearity $f$ which satisfies $(\mathrm{S})$. One can weaken the star-shaped assumption and still have uniqueness, see [22], but we do not pursue this approach here. In Section 3, we extend these results to $(P)_{\lambda}$. For more results the on uniqueness of solutions for various elliptic problems involving parameters, see [12].

For questions on the regularity of the extremal solution in fourth order problems, we direct the interested reader to [10]. We also mention the recent preprint [9] which examines the same issues as this paper but for equations of the form $\Delta^{2} u=\lambda f(u)$ in $\Omega$ with either the Dirichlet boundary conditions $u=|\nabla u|=0$ on $\partial \Omega$ or the Navier boundary conditions $u=\Delta u=0$ on $\partial \Omega$. Elliptic systems of the form $-\Delta u=\lambda f(v)$, $-\Delta v=\gamma g(u)$ in $\Omega$ with $u=v=0$ on $\partial \Omega$ are also examined.

1.2. The nonlocal eigenvalue problem. We first give the needed background regarding $(-\Delta)^{\frac{1}{2}}$ to examine $(P)_{\lambda}$, for a more detailed background see [6]. In [7] they examined the problem $(P)_{\lambda}$ with $(-\Delta)^{s}$ replacing $(-\Delta)^{\frac{1}{2}}$ and with $g(x)=1$. They 
did not investigate the questions, we are interested in but they did develop much of the needed theory to examine $(P)_{\lambda}$ and so we will use many of their results.

There are various ways to make sense of $(-\Delta)^{\frac{1}{2}} u$. Suppose that $u(x)$ is a smooth function defined in $\Omega$ which is zero on $\partial \Omega$ and suppose that $u(x)=\sum_{k} a_{k} \phi_{k}(x)$ where $\left(\phi_{k}, \lambda_{k}\right)$ are the eigenpairs of $-\Delta$ in $H_{0}^{1}(\Omega)$ which are $L^{2}$ normalized. Then one defines

$$
(-\Delta)^{\frac{1}{2}} u(x)=\sum_{k} a_{k} \sqrt{\lambda_{k}} \phi_{k}(x) .
$$

Another way is to suppose we are given $u(x)$ which is zero on $\partial \Omega$ and we let $u_{e}=$ $u_{e}(x, y)$ denote a solution of

$$
\begin{cases}\Delta u_{e}=0 & \text { in } \mathcal{C}:=\Omega \times(0, \infty) \\ u_{e}=0 & \text { on } \partial_{L} \mathcal{C}:=\partial \Omega \times(0, \infty) \\ u_{e}=u(x) & \text { in } \Omega \times\{0\}\end{cases}
$$

Then we define

$$
(-\Delta)^{\frac{1}{2}} u(x)=\left.\partial_{\nu} u_{e}(x, y)\right|_{y=0},
$$

where $\nu$ is the outward pointing normal on the bottom of the cylinder, $\mathcal{C}$. We call $u_{e}$ the harmonic extension of $u$. We define $H_{0, L}^{1}(\mathcal{C})$ to be the completion of $C_{c}^{\infty}(\Omega \times[0, \infty))$ under the norm $\|u\|^{2}:=\int_{\mathcal{C}}|\nabla u|^{2}$. When working on the cylinder generally we will write integrals of the form $\int_{\Omega \times\{y=0\}} \gamma\left(u_{e}\right)$ as $\int_{\Omega} \gamma(u)$.

Some of our results require one to examine quite weak notions of solutions to $(P)_{\lambda}$ and so we begin with our definition of a weak solution.

Definition 1. Given $h(x) \in L^{1}(\Omega)$ we say that $u \in L^{1}(\Omega)$ is a weak solution of

$$
\begin{cases}(-\Delta)^{\frac{1}{2}} u=h(x) & \text { in } \Omega \\ u=0 & \text { on } \partial \Omega,\end{cases}
$$

provided that

$$
\int_{\Omega} u \psi=\int_{\Omega} h(x)(-\Delta)^{-\frac{1}{2}} \psi \quad \forall \psi \in C_{c}^{\infty}(\Omega) .
$$

Here $(-\Delta)^{-\frac{1}{2}} \psi$ is given by the function $\phi$ where

$$
\begin{cases}(-\Delta)^{\frac{1}{2}} \phi=\psi & \text { in } \Omega \\ \phi=0 & \text { on } \partial \Omega\end{cases}
$$

The following is a weakened special case of a lemma taken from [7].

Lemma 1. Suppose that $h \in L^{1}(\Omega)$. Then there exists a unique weak solution $u$ of (1.1). Moreover if $0 \leq h$ a.e. then $u \geq 0$ in $\Omega$.

Definition 2. Let $f$ be a nonlinearity satisfying $(\mathrm{R})$.

- We say that $u(x) \in L^{1}(\Omega)$ is a weak solution of $(P)_{\lambda}$ provided $g(x) f(u) \in$ $L^{1}(\Omega)$, and

$$
\int_{\Omega} u \psi=\lambda \int_{\Omega} g(x) f(u)(-\Delta)^{-\frac{1}{2}} \psi \quad \forall \psi \in C_{c}^{\infty}(\Omega) .
$$


- We say $u$ is a regular energy solution of $(P)_{\lambda}$ provided that $u$ is bounded, the harmonic extension $u_{e}$ of $u$, is an element of $H_{0, L}^{1}(\mathcal{C})$ and satisfies

$$
\int_{\mathcal{C}} \nabla u_{e} \cdot \nabla \phi=\lambda \int_{\Omega} g(x) f(u) \phi
$$

for all $\phi \in H_{0, L}^{1}(\mathcal{C})$.

- We say $\bar{u}$ is a regular energy supersolution of $(P)_{\lambda}$ provided that $0 \leq \bar{u}$ is bounded, the harmonic extension of $\bar{u}$ is an element of $H_{0, L}^{1}(\mathcal{C})$ and satisfies

$$
\int_{\mathcal{C}} \nabla \bar{u}_{e} \cdot \nabla \phi \geq \lambda \int_{\Omega} g(x) f(\bar{u}) \phi
$$

for all $0 \leq \phi \in H_{0, L}^{1}(\mathcal{C})$.

In the case where $f$ satisfies $(\mathrm{S})$ a few minor changes are needed in the definition of solutions. For a weak solution $u$ one requires that $u \leq 1$ a.e. in $\Omega$. For $u$ to be a regular energy solution one requires that $\sup _{\Omega} u<1$.

We will need the following monotone iteration result, see [7]. Suppose that $\underline{u}$ and $\bar{u}$ are regular energy sub and supersolutions of $(P)_{\lambda}$. Then there exists a regular energy solution $u$ of $(P)_{\lambda}$ and $\underline{u} \leq u \leq \bar{u}$ in $\Omega$. By a regular energy subsolution, we are using the natural analog of regular energy supersolution.

We define the extremal parameter

$$
\lambda^{*}:=\sup \left\{0 \leq \lambda:(P)_{\lambda} \text { has a regular energy solution }\right\},
$$

and we now show some basic properties.

Lemma 2. (1) Then $0<\lambda^{*}$.

(2) Then $\lambda^{*}<\infty$.

(3) For $0<\lambda<\lambda^{*}$ there exists a regular energy solution $u_{\lambda}$ of $(P)_{\lambda}$ which is minimal and semi-stable.

(4) For each $x \in \Omega$ the map $\lambda \mapsto u_{\lambda}(x)$ is increasing on $\left(0, \lambda^{*}\right)$ and hence the pointwise limit $u^{*}(x):=\lim _{\lambda} \gamma^{*} u_{\lambda}(x)$ is well defined. Then $u^{*}$ is a weak solution of $(P)_{\lambda^{*}}$ and satisfies $\int_{\Omega} g(x) f^{\prime}\left(u^{*}\right) f\left(u^{*}\right) d x<\infty$.

In this paper, we do not need the notion of a semi-stable solution other than for the proof of (4). For the definition of a semi-stable solution one can either use a nonlocal notion, see [7] or instead work on the cylinder which is what we choose to do. We say that a regular energy solution $u$ of $(P)_{\lambda}$ is semi-stable provided that

$$
\int_{\mathcal{C}}|\nabla \phi|^{2} \geq \lambda \int_{\Omega} g(x) f(u) \phi^{2} \quad \forall \phi \in H_{0, L}^{1}(\mathcal{C}) .
$$

We now prove the lemma.

Proof: (1) Let $\bar{u}$ denote a solution of $(-\Delta)^{\frac{1}{2}} \bar{u}=\operatorname{tg}(x)$ with $\bar{u}=0$ on $\partial \Omega$ where $t>0$ is small enough such that $\sup _{\Omega} \bar{u}<1$. One sees that $\bar{u}$ is a regular energy supersolution of $(P)_{\lambda}$ provided $t \geq \lambda \sup _{\Omega} f(\bar{u})$ which clearly holds for small positive $\lambda$. Zero is clearly a regular energy subsolution and so we can apply the monotone iteration procedure to obtain a regular energy solution and hence $\lambda^{*}>0$.

(2) Suppose that either $f$ satisfies $(\mathrm{R})$ and $C_{f}<\infty$ or $f$ satisfies (S) and so trivially $C_{f}<\infty$. 
Let $u$ denote a regular energy solution of $(P)_{\lambda}$ and let $u_{e}$ denote the harmonic extension. Let $\phi$ denote the first eigenfunction of $-\Delta$ in $H_{0}^{1}(\Omega)$ and let $\phi_{e}$ be its harmonic extension; so $\phi_{e}(x, y)=\phi(x) e^{-\sqrt{\lambda_{1}} y}$. Multiply $0=-\Delta u_{e}$ by $\frac{\phi_{e}}{f\left(u_{e}\right)}$ and integrate this over the cylinder $\mathcal{C}$ to obtain

$$
\int_{\Omega} \lambda g(x) \phi=\int_{\mathcal{C}} \frac{\nabla u_{e} \cdot \nabla \phi_{e}}{f\left(u_{e}\right)}-\int_{\mathcal{C}} \frac{\left|\nabla u_{e}\right|^{2} \phi_{e} f^{\prime}\left(u_{e}\right)}{f\left(u_{e}\right)^{2}},
$$

and note that the second integral on the right is nonpositive and hence we can rewrite this as

$$
\int_{\Omega} \lambda g(x) \phi \leq \int_{\mathcal{C}} \nabla \phi_{e} \cdot \nabla h\left(u_{e}\right)
$$

where $h(t)=\int_{0}^{t} \frac{1}{f(\tau)} d \tau$. Integrating the right-hand side by parts, we have that it is equal to $\int_{\Omega}(-\Delta)^{\frac{1}{2}} \phi h(u)$ which is equal to $\sqrt{\lambda_{1}} \int_{\Omega} \phi h(u)$. So $h(u) \leq C_{f}$ and hence we have

$$
\lambda \int_{\Omega} g(x) \phi \leq \sqrt{\lambda_{1}} C_{f} \int_{\Omega} \phi .
$$

This shows that $\lambda^{*}<\infty$. The case where $f$ satisfies $(\mathrm{R})$ and where $C_{f}=\infty$ needs a separate proof, see the proof of (4). Note that there are examples of $f$ which satisfy (R) and for which $C_{f}=\infty$, for example $f(t):=(t+1) \log (t+1)+1$.

(3) The proof in the case where $g(x)=1$ also works here, see [7].

(4) Again the proof used in the case where $g(x)=1$ works to show the monotonicity of $u_{\lambda}$, see [7], and hence $u^{*}$ is well defined. One should note that our notion of a weak solution is more restrictive than what is typically used, i.e., we require $g(x) f(u) \in$ $L^{1}(\Omega)$ where typically one would only require that $\delta(x) g(x) f(u) \in L^{1}(\Omega)$ where $\delta(x)$ is the distance from $x$ to $\partial \Omega$. Hence here our proof will differ from [7].

Claim: There exist some $C<\infty$ such that

$$
\int_{\Omega} g(x) f^{\prime}\left(u_{\lambda}\right) f\left(u_{\lambda}\right) \leq C,
$$

for all $0<\lambda<\lambda^{*}$ (at this point we are allowing for the possibility of $\lambda^{*}=\infty$ ). We first show that the claim implies that $\lambda^{*}<\infty$. Note that if $(-\Delta)^{\frac{1}{2}} \phi=g(x)$ with $\phi=0$ on $\partial \Omega$ then an application of the maximum principle along with the fact that $f\left(u_{\lambda}\right) \geq 1$ gives $u_{\lambda} \geq \lambda \phi$ in $\Omega$. This along with (1.4) rules out the possibility of $\lambda^{*}=\infty$. Using a proof similar to the one in [7] one sees that $u^{*}$ is a weak solution to $(P)_{\lambda^{*}}$ except for the extra integrability condition $g(x) f\left(u^{*}\right) \in L^{1}(\Omega)$ that we require. But sending $\lambda \nearrow \lambda^{*}$ in (1.4) gives us the desired regularity and we are done.

We now prove the claim. Let $u=u_{\lambda}$ denote the minimal solution of $(P)_{\lambda}$ and let $u_{e}$ denote its harmonic extension. Take $\psi:=f\left(u_{e}\right)-1$ in (1.3) ( $\psi$ can be shown to be an admissible test function) and write the right-hand side as

$$
\int_{\mathcal{C}} \nabla\left(f\left(u_{e}\right)-1\right) f^{\prime}\left(u_{e}\right) \cdot \nabla u_{e}
$$

and integrate this by parts. Using $(P)_{\lambda}$ and after some cancellation one arrives at

$$
\int_{\mathcal{C}}\left(f\left(u_{e}\right)-1\right) f^{\prime \prime}\left(u_{e}\right)\left|\nabla u_{e}\right|^{2} \leq \lambda \int_{\Omega} g(x) f^{\prime}(u) f(u) .
$$


Define $H(t):=\int_{0}^{t} f^{\prime \prime}(\tau)(f(\tau)-1) d \tau$ and so the left-hand side of (1.5) can be written as $\int_{\mathcal{C}} \nabla H\left(u_{e}\right) \cdot \nabla u_{e}$ and integrating this by parts gives

$$
\lambda \int_{\Omega} g(x) f(u) H(u) .
$$

Combining this with (1.5) gives

$$
\int_{\Omega} g(x) f(u) H(u) \leq \int_{\Omega} g(x) f(u) f^{\prime}(u) .
$$

To complete the proof, we show that $H(u)$ dominates $f^{\prime}(u)$ for big $u$ (resp. $u$ near 1 ) when $f$ satisfies $(\mathrm{R})$ (resp. $(\mathrm{S}))$. If $0<T<t$ then one easily sees that

$$
H(t) \geq(f(T)-1)\left(f^{\prime}(t)-f^{\prime}(T)\right) .
$$

Using this along with (1.6) and dividing the domain of $\Omega$ into regions $\{u \geq T\}$ and $\{u<T\}$ one obtains the claim.

\section{Uniqueness of the extremal solution}

Theorem 1. Suppose that either $f$ satisfies $(R)$ and is $\log$ convex or satisfies $(S)$ and is strictly convex. Then the followings hold.

(1) There are no weak solutions for $(P)_{\lambda}$ for any $\lambda>\lambda^{*}$.

(2) The extremal solution $u^{*}$ is the unique weak solution of $(P)_{\lambda^{*}}$.

The following are some properties that the nonlinearity $f$ satisfies.

Proposition 1. (1) Let $f$ be a log convex nonlinearity which satisfies $(R)$.

(i) For all $0<\lambda<1$ and $\delta>0$ there exists $k>0$ such that

$$
f\left(\lambda^{-1} t\right)+k \geq(1+\delta) f(t) \text { for all } 0 \leq t<\infty .
$$

(ii) Given $\varepsilon>0$ there exists $0<\mu<1$ such that

$$
\mu^{2}\left(f\left(\mu^{-1} t\right)+\varepsilon\right) \geq f(t)+\frac{\varepsilon}{2} \text { for all } 0 \leq t<\infty .
$$

(iii) Then $f$ is strictly convex.

(2) Let $f$ be a nonlinearity which satisfies $(S)$.

(i) Given $\varepsilon>0$ there exists $0<\mu<1$ such that

$$
\mu\left(f\left(\mu^{-1} t\right)+\varepsilon\right) \geq f(t)+\frac{\varepsilon}{2} \quad \text { for all } \quad 0 \leq t \leq \mu .
$$

(ii) Then $\lim _{t \nearrow 1} \frac{f(t)}{F(t)}=\infty$ where $F(t):=\int_{0}^{t} f(\tau) d \tau$.

Proof. See $[1,17]$ for the proof of (1)-(i) and (1)-(ii). Part (1)-(iii) is trivial.

(2)-(i) Set $h(t):=\mu\left\{f\left(\mu^{-1} t\right)+\varepsilon\right\}-f(t)-\frac{\varepsilon}{2}$ and note that $h^{\prime}(t) \geq 0$ for all $0 \leq t \leq \mu$ and that $h(0)>0$ for $\mu$ sufficiently close to 1 , which gives us the desired result.

(2)-(ii) Let $0<t<1$ and we use a Riemann sum with right-hand endpoints to approximate $F(t)$. So for any positive integer $n$ we have

$$
F(t) \leq \frac{t}{n} \sum_{k=1}^{n} f\left(\frac{k t}{n}\right) \leq \frac{t(n-1)}{n} f\left(\frac{(n-1) t}{n}\right)+\frac{t}{n} f(t),
$$


and so

$$
\limsup _{t \nearrow 1} \frac{F(t)}{f(t)} \leq \frac{1}{n}
$$

but since $n$ is arbitrary we have the desired result.

The following is an essential step in proving Theorem 1 . We give the proof of this lemma later.

Lemma 3. Suppose that $f$ is log convex and satisfies $(R)$ or $f$ satisfies (S). Suppose $\varepsilon>0$ and that $0 \leq \tau$ is a weak solution of

$$
\begin{cases}(-\Delta)^{\frac{1}{2}} \tau=l(x) & \text { in } \Omega \\ \tau=0 & \text { on } \partial \Omega\end{cases}
$$

where $g(x)(f(\tau)+\varepsilon) \leq l(x) \in L^{1}(\Omega)$. Then there exists a regular energy solution of

$$
\begin{cases}(-\Delta)^{\frac{1}{2}} u=g(x)\left(f(u)+\frac{\varepsilon}{2}\right) & \text { in } \Omega \\ u=0 & \text { on } \partial \Omega .\end{cases}
$$

Proof of Theorem 1: Without loss of generality assume that $\lambda^{*}=1$ and let $u^{*}$ denote the extremal solution of $(P)_{\lambda^{*}}$. Suppose that $v$ is also a weak solution of $(P)_{\lambda^{*}}$ and $v$ is not equal to $u^{*}$. Set $\Omega_{0}:=\left\{x \in \Omega: u^{*}(x) \neq v(x), u^{*}(x), v(x) \in \mathbb{R}\right\}$ (resp. $\Omega_{0}=\left\{x \in \Omega: u^{*}(x) \neq v(x), u^{*}(x), v(x)<1\right\}$ ) when $f$ satisfies (R) (resp. (S)) and note that $\left|\Omega_{0}\right|>0$. Define

$$
h(x):= \begin{cases}\frac{f\left(u^{*}(x)\right)+f(v(x))}{2}-f\left(\frac{u^{*}(x)+v(x)}{2}\right) & x \in \Omega_{0} \\ 0 & \text { otherwise. }\end{cases}
$$

Note that by the strict convexity of $f$, which we obtain either by hypothesis or by Proposition 1, we have $0 \leq h$ in $\Omega$ and $h>0$ in $\Omega_{0}$. Also note that $h \in L^{1}(\Omega)$. Define $z:=\frac{u^{*}+v}{2}$. Since $u^{*}$ and $v$ are weak solutions of $(P)_{\lambda^{*}}, z$ is a weak solution of

$$
(-\Delta)^{\frac{1}{2}} z=g(x) f(z)+g(x) h(x) \text { in } \Omega,
$$

with $z=0$ on $\partial \Omega$. From now on we omit the boundary values since they will always be zero unless otherwise mentioned. Let $\chi$ and $\phi$ denote weak solutions of $(-\Delta)^{\frac{1}{2}} \chi=$ $g(x) h(x)$ and $(-\Delta)^{\frac{1}{2}} \phi=g(x)$ in $\Omega$. By taking $\varepsilon>0$ small enough one has that $\chi \geq \varepsilon \phi$ in $\Omega$. Set $\tau:=z+\varepsilon \phi-\chi$ and note that $\tau$ is a weak solution of

$$
(-\Delta)^{\frac{1}{2}} \tau=g(x)(f(z)+\varepsilon) \geq 0 \quad \text { in } \Omega,
$$

and by Lemma 1 , we have that $0 \leq \tau$. Moreover, from the fact that $\tau \leq z$ in $\Omega$ we have

$$
g(x)(f(\tau)+\varepsilon) \leq(-\Delta)^{\frac{1}{2}} \tau \in L^{1}(\Omega) .
$$

Applying Lemma 3, there exists a regular energy solution $u$ of

$$
(-\Delta)^{\frac{1}{2}} u=g(x)\left(f(u)+\frac{\varepsilon}{2}\right) \quad \text { in } \Omega .
$$

Set $w:=u+\alpha u-\frac{\varepsilon}{2} \phi$ where $\alpha>0$ is chosen small enough such that $\alpha u \leq \frac{\varepsilon}{2} \phi$ in $\Omega$. A straightforward computation shows that $w$ is a regular energy solution of

$$
(-\Delta)^{\frac{1}{2}} w=(1+\alpha) g(x) f(u)+\frac{\varepsilon}{2} \alpha g(x) \quad \text { in } \Omega
$$


and that $w \leq u$ in $\Omega$. By Lemma 1 , we also have $0 \leq w$ in $\Omega$. From this, we see that $w$ is a regular energy supersolution of

$$
(-\Delta)^{\frac{1}{2}} w \geq(1+\alpha) g(x) f(w) \text { in } \Omega,
$$

with zero boundary conditions. We now apply the monotone iteration argument to obtain a regular energy solution $\tilde{u}$ of $(-\Delta)^{\frac{1}{2}} \tilde{u}=(1+\alpha) g(x) f(\tilde{u})$ in $\Omega$ which contradicts the fact that $\lambda^{*}=1$. So, we have shown that $\left|\Omega_{0}\right|=0$ and so $u^{*}=v$ a.e. in $\Omega$.

Proof of Lemma 3: Let $\varepsilon>0$ and suppose that $0 \leq \tau \in L^{1}(\Omega)$ is a weak solution of $(-\Delta)^{\frac{1}{2}} \tau=l(x)$ in $\Omega$ where $0 \leq g(x)(f(\tau)+\varepsilon) \leq l(x)$ in $\Omega$. As in the proof of Theorem 1 , we omit the boundary values since they will always be Dirichlet boundary conditions and we also assume that $\lambda^{*}=1$. First, assume that $f$ is a $\log$ convex nonlinearity, which satisfies $(\mathrm{R})$. Let $u_{0}:=\tau$ and let $u_{1}, u_{2}, u_{3}$ be weak solutions of

$$
\begin{array}{ll}
(-\Delta)^{\frac{1}{2}} u_{1}=\mu g(x)\left(f\left(u_{0}\right)+\varepsilon\right) & \text { in } \Omega, \\
(-\Delta)^{\frac{1}{2}} u_{2}=\mu g(x)\left(f\left(u_{1}\right)+\varepsilon\right) & \text { in } \Omega, \\
(-\Delta)^{\frac{1}{2}} u_{3}=\mu g(x)\left(f\left(u_{2}\right)+\varepsilon\right) & \text { in } \Omega,
\end{array}
$$

where $0<\mu<1$ is the constant given in Proposition 1 such that $\mu^{2}\left(f\left(\frac{t}{\mu}\right)+\varepsilon\right) \geq$ $f(t)+\frac{\varepsilon}{2}$ for all $t \geq 0$. One easily sees that $u_{2} \leq u_{1} \leq \mu u_{0}$. Now note that

$$
\begin{aligned}
(-\Delta)^{\frac{1}{2}} u_{1} & =\mu g(x)\left(f\left(u_{0}\right)+\varepsilon\right) \\
& \geq \mu g(x)\left(f\left(\frac{u_{1}}{\mu}\right)+\varepsilon\right) .
\end{aligned}
$$

By Proposition 1 with $\delta:=2 N-1>0$ and $0<\lambda=\mu<1$ there exist some $k>0$ such that

hence one can rewrite $(2.1)$ as

$$
f\left(\frac{u_{1}}{\mu}\right) \geq 2 N f\left(u_{1}\right)-k
$$

$$
(-\Delta)^{\frac{1}{2}} u_{1} \geq \mu g(x)\left(2 N f\left(u_{1}\right)-k+\varepsilon\right) .
$$

We let $\phi$ be as in the proof of Theorem 1 and examine $u_{1}+t \phi$ where $t>0$ is to be picked later. Note that

$$
\begin{aligned}
(-\Delta)^{\frac{1}{2}}\left(u_{1}+t \phi\right) & =(-\Delta)^{\frac{1}{2}} u_{1}+t g(x) \\
& \geq 2 N \mu g(x)\left(f\left(u_{1}\right)+\varepsilon\right)+m g(x),
\end{aligned}
$$

where $m:=t-\mu k+\varepsilon \mu(1-2 N)$ and we now pick $t>0$ big enough such that $m=0$. Therefore, from the definition of $u_{2}$ we have that

$$
(-\Delta)^{\frac{1}{2}}\left(u_{1}+t \phi\right) \geq 2 N(-\Delta)^{\frac{1}{2}} u_{2} \quad \text { in } \Omega .
$$

So, from the maximum principle we obtain

$$
u_{2} \leq \frac{1}{2 N}\left(u_{1}+t \phi\right) \text { in } \Omega .
$$

Since $f$ is log convex, there is some smooth, convex increasing function $\beta$ with $\beta(0)=0$ and $f(t)=e^{\beta(t)}$. By the convexity of $\beta$ and since $\beta(0)=0$, we have

$$
\beta\left(u_{2}\right) \leq \frac{1}{2 N} \beta\left(u_{1}+t \phi\right) \leq \frac{1}{2 N} \beta\left(\mu u_{0}+t \phi\right),
$$


but

$$
\beta\left(\mu u_{0}+t \phi\right)=\beta\left(\mu u_{0}+(1-\mu) \frac{t \phi}{1-\mu}\right) \leq \mu \beta\left(u_{0}\right)+(1-\mu) \beta\left(\frac{t \phi}{1-\mu}\right) .
$$

From this, we can conclude

$$
f\left(u_{2}\right)^{2 N} \leq e^{\mu \beta\left(u_{0}\right)} e^{(1-\mu) \beta\left(\frac{t \phi}{1-\mu}\right)} \leq f\left(u_{0}\right) f\left(\frac{t \phi}{1-\mu}\right)^{1-\mu} .
$$

So, we see that $g(x) f\left(u_{2}\right)^{2 N} \leq C g(x) f\left(u_{0}\right) \in L^{1}(\Omega)$ for some large constant $C$.

Since $g(x)$ is bounded, we conclude that $g(x) f\left(u_{2}\right) \in L^{2 N}(\Omega)$. But $u_{3}$ satisfies $(-\Delta)^{\frac{1}{2}} u_{3}=\mu g(x)\left(f\left(u_{2}\right)+\varepsilon\right)$ in $\Omega$ and so by elliptic regularity we have that $u_{3}$ is bounded (since the right-hand side is an element of $L^{p}(\Omega)$ for some $p>N$ ) and now we use the fact that $0 \leq u_{3} \leq u_{2}$ and the monotone iteration argument to obtain a regular energy solution $w$ to $(-\Delta)^{\frac{1}{2}} w=\mu g(x)(f(w)+\varepsilon)$ in $\Omega$.

Now, set $\xi:=\mu w$ and note that $\xi$ is a regular energy solution of

$$
(-\Delta)^{\frac{1}{2}} \xi=\mu^{2} g(x)\left(f\left(\frac{\xi}{\mu}\right)+\varepsilon\right) \quad \text { in } \Omega,
$$

and from Proposition 1, we have

$$
(-\Delta)^{\frac{1}{2}} \xi \geq g(x)\left(f(\xi)+\frac{\varepsilon}{2}\right) \quad \text { in } \Omega
$$

and so by an iteration argument, we have the desired result.

Now, assume that $f$ satisfies $(\mathrm{S})$. In this case, the proof is much simpler. Define $w:=\mu \tau$ where $0<\mu<1$ is from Proposition 1 . Then note that $0 \leq w \leq \mu$ a.e. and

$$
\begin{aligned}
(-\Delta)^{\frac{1}{2}} w=\mu l(x) & \geq \mu g(x)\left(f\left(\frac{w}{\mu}\right)+\varepsilon\right) \\
& \geq g(x)\left(f(w)+\frac{\varepsilon}{2}\right) .
\end{aligned}
$$

Hence, $w$ is a regular energy supersolution of

$$
(-\Delta)^{\frac{1}{2}} w \geq g(x)\left(f(w)+\frac{\varepsilon}{2}\right),
$$

and we have the desired result after an application of the monotone iteration argument.

\section{Uniqueness of solutions for small $\lambda$}

In this section, we prove uniqueness theorems for equation $(P)_{\lambda}$ for small enough $\lambda$. Throughout this section, we assume that $g=0$ on $\partial \Omega$. We need the following regularity result.

Proposition 2. [6] Let $\alpha \in(0,1), \Omega$ be a $C^{2, \alpha}$ bounded domain in $\mathbb{R}^{N}$ and suppose that $u$ is a weak solution of $(-\Delta)^{\frac{1}{2}} u=h(x)$ in $\Omega$ with $u=0$ on $\partial \Omega$.

(1) Suppose that $h \in L^{\infty}(\Omega)$. Then $u_{e} \in C^{0, \alpha}(\overline{\mathcal{C}})$ hence $u \in C^{0, \alpha}(\bar{\Omega})$.

(2) Suppose that $h \in C^{k, \alpha}(\bar{\Omega})$ where $k=0$ or $k=1$ and $h=0$ on $\partial \Omega$. Then $u_{e} \in C^{k+1, \alpha}(\overline{\mathcal{C}})$ hence $u \in C^{k+1, \alpha}(\bar{\Omega})$. 
Using this one easily obtains the following:

Corollary 1. For each $0<\lambda<\lambda^{*}$ the minimal solution of $(P)_{\lambda}$, $u_{\lambda}$, belongs to $C^{2, \alpha}(\bar{\Omega})$. In addition $u_{\lambda} \rightarrow 0$ in $C^{1}(\bar{\Omega})$ as $\lambda \rightarrow 0$.

We now come to our main theorem of this section.

Theorem 2. Suppose that $\Omega$ is a star-shaped domain with respect to the origin and set $\gamma:=\sup _{\Omega} \frac{x \cdot \nabla g(x)}{g(x)}$.

(1) Suppose that $f$ satisfies $(R)$ and that

$$
\limsup _{t \rightarrow \infty} \frac{F(t)}{f(t) t}<\frac{N-1}{2(N+\gamma)} .
$$

Then for sufficiently small $\lambda, u_{\lambda}$ is the unique regular energy solution of $(P)_{\lambda}$.

(2) Suppose that $f$ satisfies $(S)$. Then for sufficiently small $\lambda, u_{\lambda}$ is the unique regular energy solution $(P)_{\lambda}$.

Proof: Let $f$ satisfy (R) and (3.1) or let $f$ satisfy (S) and suppose that $u$ is a second regular energy solution of $(P)_{\lambda}$ which is different from the minimal solution $u_{\lambda}$. Set $v:=u-u_{\lambda}$ and note that $v \geq 0$ by the minimality of $u_{\lambda}$ and $v \neq 0$ since $u$ is different from the minimal solution.

A computation shows that $v$ satisfies the equation

$$
(-\Delta)^{\frac{1}{2}} v=\lambda g(x)\left\{f\left(u_{\lambda}+v\right)-f\left(u_{\lambda}\right)\right\} .
$$

Applying Proposition 2 to $u$ and $u_{\lambda}$ separately shows that $v_{e} \in C^{2, \alpha}(\overline{\mathcal{C}})$.

A computation shows the following identity holds:

$$
\operatorname{div}\left\{\left(z, \nabla v_{e}\right) \nabla v_{e}-z \frac{\left|\nabla v_{e}\right|^{2}}{2}\right\}+\frac{N-1}{2}\left|\nabla v_{e}\right|^{2}=\left(z, \nabla v_{e}\right) \Delta v_{e}
$$

where $z=(x, y)$. Integrating this identity over $\Omega \times(0, R)$ we end up with

$$
\frac{1}{2} \int_{\partial \Omega \times(0, R)}\left|\nabla v_{e}\right|^{2} x \cdot \nu+\int_{\Omega} x \cdot \nabla_{x} v_{e} \partial_{\nu} v_{e}+\frac{N-1}{2} \int_{\Omega \times(0, R)}\left|\nabla v_{e}\right|^{2}+\varepsilon(R)=0
$$

where

$$
\varepsilon(R):=\int_{\Omega \times\{y=R\}}\left(x \cdot \nabla_{x} v_{e}+R \partial_{y} v_{e}\right) \partial_{y} v_{e}-\frac{R}{2}\left|\nabla v_{e}\right|^{2} .
$$

One can show that $\varepsilon(R) \rightarrow 0$ as $R \rightarrow \infty$, for details on this and the above calculations see [24]. Sending $R \rightarrow \infty$ and since $\Omega$ is star-shaped with respect to the origin, we have

$$
\frac{N-1}{2} \int_{\mathcal{C}}\left|\nabla v_{e}\right|^{2} \leq-\int_{\Omega} x \cdot \nabla_{x} v \partial_{\nu} v_{e}
$$

and after using (3.2) one obtains

$$
\frac{N-1}{2} \int_{\mathcal{C}}\left|\nabla v_{e}\right|^{2} \leq-\lambda \int_{\Omega} x \cdot \nabla_{x} v g(x)\left\{f\left(u_{\lambda}+v\right)-f\left(u_{\lambda}\right)\right\} .
$$

We now compute the right-hand side of (3.4). Set $h(x, \tau):=f\left(u_{\lambda}(x)+\tau\right)-f\left(u_{\lambda}(x)\right)$ and let $H(x, t)=\int_{0}^{t} h(x, \tau) d \tau$. For this portion of the proof, we are working on $\Omega$ and 
hence all gradients are with respect to the $x$-variable. To clarify our notation note that the chain rule can be written as

$$
\nabla H(x, v)=\nabla_{x} H(x, v)+h(x, v) \nabla v,
$$

where we recall $v=v(x)$. Some computations now show that

$$
H(x, t)=F\left(u_{\lambda}+t\right)-F\left(u_{\lambda}\right)-f\left(u_{\lambda}\right) t,
$$

and

$$
\nabla_{x} H(x, t)=\left\{f\left(u_{\lambda}+t\right)-f\left(u_{\lambda}\right)-f^{\prime}\left(u_{\lambda}\right) t\right\} \nabla u_{\lambda},
$$

and so the right-hand side of (3.4) can be written as

$$
\begin{aligned}
-\lambda \int_{\Omega} g(x)\left\{f\left(u_{\lambda}+v\right)-f\left(u_{\lambda}\right)\right\} x \cdot \nabla v & =-\lambda \int_{\Omega} g(x) h(x, v) x \cdot \nabla v \\
= & -\lambda \int_{\Omega} g(x) x \cdot\left\{\nabla H(x, v)-\nabla_{x} H(x, v)\right\} \\
= & \lambda \int_{\Omega} g(x) x \cdot \nabla_{x} H(x, v)+\lambda N \int H(x, v) g(x) \\
& +\lambda \int_{\Omega} H(x, v) x \cdot \nabla g(x) .
\end{aligned}
$$

Therefore, (3.4) can be written as

$$
\begin{aligned}
\frac{N-1}{2} \int_{\mathcal{C}}\left|\nabla v_{e}\right|^{2} \leq & \lambda \int_{\Omega} x \cdot \nabla u_{\lambda} g(x)\left\{f\left(u_{\lambda}+v\right)-f\left(u_{\lambda}\right)-f^{\prime}\left(u_{\lambda}\right) v\right\} \\
& +N \lambda \int_{\Omega} g(x)\left\{F\left(u_{\lambda}+v\right)-F\left(u_{\lambda}\right)-f\left(u_{\lambda}\right) v\right\} \\
& +\lambda \int_{\Omega} x \cdot \nabla g(x)\left\{F\left(u_{\lambda}+v\right)-F\left(u_{\lambda}\right)-f\left(u_{\lambda}\right) v\right\} .
\end{aligned}
$$

We now assume that we are in case (1). Let $\alpha$ be such that

$$
\limsup _{\tau \rightarrow \infty} \frac{F(\tau)}{\tau f(\tau)}<\alpha<\frac{N-1}{2(N+\gamma)},
$$

so there exist some $\tau_{0}>0$ such that $F(\tau)<\alpha \tau f(\tau)$ for all $\tau \geq \tau_{0}$. Let $0<\theta<1$ be such that $\frac{\theta(N-1)}{2}-\alpha(N+\gamma)>0$ and we now decompose the left-hand side of (3.5) into the convex combination

$$
\frac{\theta(N-1)}{2} \int_{\mathcal{C}}\left|\nabla v_{e}\right|^{2}+\frac{(N-1)(1-\theta)}{2} \int_{\mathcal{C}}\left|\nabla v_{e}\right|^{2}
$$

Using the following trace theorem: there exist some $\tilde{C}>0$ such that

$$
\int_{\mathcal{C}}|\nabla w|^{2} \geq \tilde{C} \int_{\Omega} w^{2}, \quad \forall w \in H_{0, L}^{1}(\mathcal{C}),
$$

one sees that (3.6) is bounded below by

$$
\frac{\theta(N-1)}{2} \int_{\mathcal{C}}\left|\nabla v_{e}\right|^{2}+C \int_{\Omega} v^{2}
$$


By taking $C>0$ smaller if necessary one can bound this from below by

$$
\frac{\theta(N-1)}{2} \int_{\mathcal{C}}\left|\nabla v_{e}\right|^{2}+C \int_{\Omega} g(x) v^{2},
$$

and after using (3.2), this last quantity is equal to

$$
\frac{\lambda \theta(N-1)}{2} \int_{\Omega} g(x)\left\{f\left(u_{\lambda}+v\right)-f\left(u_{\lambda}\right)\right\} v+C \int_{\Omega} g(x) v^{2} .
$$

Substituting (3.8) into (3.4) and rearranging one arrives at an inequality of the form

$$
\int_{\Omega} g(x) T_{\lambda}(x, v) \leq 0
$$

where

$$
\begin{aligned}
T_{\lambda}(x, \tau)= & \frac{\theta(N-1)}{2}\left\{f\left(u_{\lambda}+\tau\right)-f\left(u_{\lambda}\right)\right\} \tau+\frac{C}{\lambda} \tau^{2} \\
& -N\left\{F\left(u_{\lambda}+\tau\right)-F\left(u_{\lambda}\right)-f\left(u_{\lambda}\right) \tau\right\} \\
& -\frac{x \cdot \nabla g}{g}\left\{F\left(u_{\lambda}+\tau\right)-F\left(u_{\lambda}\right)-f\left(u_{\lambda}\right) \tau\right\} \\
& -x \cdot \nabla u_{\lambda}\left\{f\left(u_{\lambda}+\tau\right)-f\left(u_{\lambda}\right)-f^{\prime}\left(u_{\lambda}\right) \tau\right\} .
\end{aligned}
$$

To obtain a contradiction, we show that for sufficiently small $\lambda>0$ that $T_{\lambda}(x, \tau)>0$ on $(x, \tau) \in \Omega \times(0, \infty)$ and hence we must have that $v=0$. Define

$$
\begin{aligned}
S_{\lambda}(x, \tau)= & \frac{\theta(N-1)}{2}\left\{f\left(u_{\lambda}+\tau\right)-f\left(u_{\lambda}\right)\right\} \tau+\frac{C}{\lambda} \tau^{2} \\
& -(N+\gamma)\left\{F\left(u_{\lambda}+\tau\right)-F\left(u_{\lambda}\right)-f\left(u_{\lambda}\right) \tau\right\} \\
& -\varepsilon_{\lambda}\left\{f\left(u_{\lambda}+\tau\right)-f\left(u_{\lambda}\right)-f^{\prime}\left(u_{\lambda}\right) \tau\right\} .
\end{aligned}
$$

where $\varepsilon_{\lambda}:=\left\|\nabla u_{\lambda} \cdot x\right\|_{L^{\infty}}$. Note that since $f$ is increasing and convex that $T_{\lambda}(x, \tau) \geq$ $S_{\lambda}(x, \tau)$ for all $\tau \geq 0$. We now show the desired positivity for $S_{\lambda}$ and to do this we examine large and small $\tau$ separately.

Large $\tau$ : Take $\tau \geq \tau_{0}$ and $0<\lambda \leq \frac{\lambda^{*}}{2}$. Since $f$ is convex and increasing

$$
\begin{aligned}
S_{\lambda}(x, \tau) \geq & \frac{\theta(N-1)}{2} f\left(u_{\lambda}+\tau\right) \tau-(N+\gamma) F\left(u_{\lambda}+\tau\right) \\
& -\varepsilon_{\lambda} f\left(u_{\lambda}+\tau\right)+\frac{C}{\lambda} \tau^{2} \\
& -\frac{\theta(N-1)}{2} f\left(u_{\lambda}\right) \tau
\end{aligned}
$$

but $F\left(u_{\lambda}+\tau\right)<\alpha\left(u_{\lambda}+\tau\right) f\left(u_{\lambda}+\tau\right)$ for all $\tau \geq \tau_{0}$ and so the right-hand side of (3.9) is bounded below by

$$
\begin{aligned}
& f\left(u_{\lambda}+\tau\right)\left[\tau\left\{\frac{\theta(N-1)}{2}-(N+\gamma) \alpha\right\}-\varepsilon_{\lambda}-(N+\gamma) \alpha u_{\lambda}\right] \\
& -\frac{\theta(N-1)}{2} f\left(u_{\lambda}\right) \tau+\frac{C}{\lambda} \tau^{2} .
\end{aligned}
$$


Using the fact that $f$ is superlinear at $\infty$ there exist some $\tau_{1} \geq \tau_{0}$ such that $S_{\lambda}(x, \tau)>$ 0 for all $\tau \geq \tau_{1}$ and $0<\lambda \leq \frac{\lambda^{*}}{2}$.

Small $\tau$ : Let $0<\lambda_{0}<\frac{\lambda^{*}}{2}$ be such that $\left\|u_{\lambda}\right\|_{L^{\infty}} \leq 1$. Using the convexity and monotonicity of $f$ and Taylor's Theorem there exist some $C_{1}>0$ such that

$$
F\left(u_{\lambda}+\tau\right)-F\left(u_{\lambda}\right)-f\left(u_{\lambda}\right) \tau \leq C_{1} \tau^{2}, \quad f\left(u_{\lambda}+\tau\right)-f\left(u_{\lambda}\right)-f^{\prime}\left(u_{\lambda}\right) \tau \leq C_{1} \tau^{2},
$$

for all $0 \leq \tau \leq \tau_{0}, 0<\lambda \leq \lambda_{0}$ and $x \in \Omega$. Noting that the first term of $S_{\lambda}(x, \tau)$ is positive for $\tau>0$ one sees that for all $0<\tau \leq \tau_{0}, x \in \Omega$ and $0<\lambda<\lambda_{0}$ one has the lower bound

$$
S_{\lambda}(x, \tau) \geq \frac{C}{\lambda} \tau^{2}-\left(N+\gamma+\varepsilon_{\lambda}\right) C_{1} \tau^{2},
$$

and hence by taking $\lambda$ smaller if necessary we have the desired result.

(2) We now assume that $f$ satisfies $(\mathrm{S})$. One uses a similar approach to arrive at an inequality of the form

$$
\int_{\Omega} T_{\lambda}(x, v) \leq 0
$$

where as before $v=u-u_{\lambda} \geq 0$ and where we assume that $v \neq 0$. To arrive at a contradiction we show that for sufficiently small $\lambda$ that $T_{\lambda}(x, \tau)>0$ for all $x \in \Omega$ and for all $0<\tau<1-u_{\lambda}(x)$. Again the idea is to break the interval into two regions. For $\tau$ such that $\tau+u_{\lambda}(x)$ close to 1 , we use Proposition 1, 2 (ii) to see the desired positivity. For the remainder of the interval, we again use Taylor's Theorem.

\section{References}

[1] E. Berchio, F. Gazzola, Some remarks on biharmonic elliptic problems with positive, increasing and convex nonlinearities. Electron. J. Differ. Equ. 34 (2005), 1-20.

[2] H. Brezis, T. Cazenave, Y. Martel, and A. Ramiandrisoa, Blow up for $u_{t}-\Delta u=g(u)$ revisited, Adv. Differ. Equ., 1 (1996), 73-90.

[3] H. Brezis and L. Vazquez, Blow-up solutions of some nonlinear elliptic problems, Rev. Mat. Univ. Complut. Madrid 10 (2) (1997), 443-469.

[4] X. Cabré, Regularity of minimizers of semilinear elliptic problems up to dimension four, Comm. Pure Appl. Math. 63 (10) (2010), 1362-1380.

[5] X. Cabré and A. Capella, Regularity of radial minimizers and extremal solutions of semilinear elliptic equations, J. Funct. Anal. 238 (2006), 709-733.

[6] X. Cabré and J. Tan, Positive solutions of nonlinear problems involving the square root of the Laplacian, Adv. Math. 224(5) (2010), 2052-2093.

[7] A. Capella, J. Dávila, L. Dupaigne, and Y. Sire, Regularity of radial extremal solutions for some non local semilinear equations. Comm. Partial Differ. Equ. 36 (2011), 1353-1384.

[8] D. Cassani, J.M. do O, and N. Ghoussoub, On a fourth order elliptic problem with a singular nonlinearity, Adv. Nonlinear Stud., 9 (2009) 177-197.

[9] C. Cowan, Uniqueness of solutions for elliptic systems and fourth order equations involving a parameter, preprint, 2011.

[10] C. Cowan, P. Esposito, and N. Ghoussoub, Regularity of extremal solutions in fourth order nonlinear eigenvalue problems on general domains. Discrete Contin. Dyn. Syst. 28(3) (2010), 1033-1050.

[11] J. Dávila, L. Dupaigne, I. Guerra, and M. Montenegro, Stable solutions for the bilaplacian with exponential nonlinearity, SIAM J. Math. Anal., 39(2) (2007), 565-592.

[12] J. Dolbeault and R. Stanćzy, Non-existence and uniqueness results for supercritical semilinear elliptic equations, Ann. Henri Poincaré 10(7) (2010), 1311-1333.

[13] P. Esposito and N. Ghoussoub, Uniqueness of solutions for an elliptic equation modeling MEMS. Methods Appl. Anal. 15(3) (2008), 341-353. 
[14] P. Esposito, N. Ghoussoub, and Y. Guo, Compactness along the branch of semi-stable and unstable solutions for an elliptic problem with a singular nonlinearity, Comm. Pure Appl. Math. 60 (2007), 1731-1768.

[15] P. Esposito, N. Ghoussoub, and Y. Guo; Mathematical Analysis of Partial Differential Equations Modeling Electrostatic MEMS, Research Monograph, Courant Lecture Notes in Mathematics, 20. Courant Institute of Mathematical Sciences, New York; American Mathematical Society, Providence, RI, 2010. xiv +318 pp.

[16] N. Ghoussoub and Y. Guo, On the partial differential equations of electro MEMS devices: stationary case, SIAM J. Math. Anal. 38 (2007), 1423-1449.

[17] X. Luo, Uniqueness of weak extremal solution to biharmonic equation with logarithmically convex nonlinearities, J. Partial Differ. Equ. 23 (2010), 315-329.

[18] Y. Martel, Uniqueness of weak extremal solutions of nonlinear elliptic problems, Houston J. Math. 23(1) (1997), 161-168.

[19] J. McGough, On solution continua of supercritical quasilinear elliptic problems, Differ. Integral Equ. 7(5-6) (1994), 1453-1471.

[20] F. Mignot and J-P. Puel, Sur une classe de problemes non lineaires avec non linearite positive, croissante, convexe, Comm. Partial Differ. Equ. 5 (1980), 791-836.

[21] G. Nedev, Regularity of the extremal solution of semilinear elliptic equations, C. R. Acad. Sci. Paris Sir. I Math. 330(11) (2000), 997-1002.

[22] R. Schaaf, Uniqueness for semilinear elliptic problems: supercritical growth and domain geometry, Adv. Differ. Equ. 5(10-12) (2000), 1201-1220.

[23] K. Schmitt, Positive solutions of semilinear elliptic boundary value problems, Topological methods in differential equations and inclusions (Montreal, PQ, 1994), NATO Adv. Sci. Inst. Ser. C Math. Phys. Sci., vol. 472, Kluwer Academic Publishers, Dordrecht, 1995, pp. 447-500.

[24] J. Tan, The Brezis-Nirenberg type problem involving the square root of the laplacian, Calc. Var. 42 (2011), 21-41.

Department of Mathematical Sciences, University of Alabama in Huntsville, 258A Shelby Center, Huntsville, AL 35899

E-mail address: ctc0013@uah.edu

Department of Mathematics, University of British Columbia, Vancouver, B.C. Canada V6T 122

E-mail address: fazly@math.ubc.ca 